\title{
Effects of Maneuvers on Breathing-Swallowing Coordination and Swallowing Kinetics in Dysphagia Patients
}

Kubo S1, Marui M1, Yagi N2,3,4, Nagami S 5,6, Hojo $\mathrm{K}^{1}$, Kunieda $\mathrm{K}^{1}$, Shigematsu $\mathbf{T}^{1}$, Kanazawa $\mathbf{H}^{1}$, Fujishima $I^{1}$ and Oku $\mathbf{Y}^{2,5 *}$

${ }^{1}$ Department of Rehabilitation, Hamamatsu City

Rehabilitation Hospital, J apan

${ }^{2}$ Department of Swallowing Physiology, Hyogo College of Medicine, Japan

${ }^{3}$ Department of Neurology, Graduate School of Medicine, Kyoto University, J apan

${ }^{4}$ Clinical Research Center for Medical Equipment Development, Kyoto University Hospital, J apan

${ }^{5}$ Department of Physiology, Hyogo College of Medicine, Japan

${ }^{6}$ Department of Sensory Science, Kawasaki University of Medical Welfare, Japan

*Correspondling author: Oku Y, Department of Physiology, Hyogo College of Medicine, 1-1 Mukogawacho, Nishinomiya, Hyogo, 663-8501, J apan

Received: October 03, 2017; Accepted: October 24, 2017; Published: October 31, 2017

\begin{abstract}
Objective: We monitored swallows before and after the 'humming swallow' and the 'forehead exercise' in dysphagia patients and evaluated whether these maneuvers were effective.

Design: For the 'humming swallow', a modified version of the supraglottic swallow, the subject was instructed to inhale and hum before a swallow. For the forehead exercise, the subject was instructed to place his palm firmly on his forehead and push his forehead against his hand strongly. Each patient performed the maneuvers sequentially. The breathing-swallowing coordination and kinetics of swallowing were measured before and after each maneuver and during the humming swallow.
\end{abstract}

Results: Choking was observed in 13 of 30 patients before the forehead exercise but only observed in two patients after the maneuver. The frequencies of inspiration before swallowing and inspiration after swallowing were greater when swallowing was accompanied by choking. In the humming swallow, the frequency of inspiration after swallowing tended to increase.

Conclusion: None of the parameters associated with swallowing kinetics showed significant changes during the two maneuvers. The humming swallow maneuver may increase the frequency of inspiration after swallow, so caution should be exercised when this technique is applied to patients with food residues in the pharynx.

Keywords: Deglutition disorders; Supraglottic swallow; Humming swallow; Forehead exercise; Swallowing kinetics; Breathing-swallowing coordination

\section{Abbreviations}

E-SW: Expiration-Swallow; I-SW: Inspiration-Swallow; SW-E: Swallow-Expiration; SW-I: Swallow-Inspiration; LRT: Laryngeal rising time; LAD: Laryngeal activation duration

\section{Introduction}

Respiration after swallowing is usually followed by expiration, which could be an airway protective mechanism, as expiration can prevent the inhalation of residual material left in the pharynx after swallowing [1]. Indeed, it has been reported that Parkinson's disease patients with decreased swallowing safety, as measured by the penetration-aspiration scale, tend to inspire after swallows (SWI pattern) and tend to have shorter swallowing apnea duration [2]. Recently, we developed a swallowing monitoring device that can detect the laryngeal motion and evaluate the coordination between breathing and swallowing [3]. Comparing the kinetics of swallowing between healthy elderly subjects and patients with dysphagia, we found that the prolongation of 'swallowing latency' (interval between the onset of respiratory pause and the onset of swallowing) leads to delayed timing of the swallow and results in an increase in the SW-I pattern in patients with dysphagia [4].

Here, we evaluate the effects of two new maneuvers by analyzing the coordination between breathing and swallowing and the timing of swallowing within the respiratory cycle. The first maneuver is the 'humming swallow', a modified version of the supraglottic swallow maneuver. In the supraglottic swallow maneuver, each subject is instructed to inhale and hold his breath before and during swallowing and to exhale after swallowing [5]. Therefore, the subject is expected to swallow at a high lung volume and naturally resume his respiration with expiration after swallowing, which would reduce the chance of aspiration. However, it is difficult to objectively confirm when someone is holding their breath, and thus, it is difficult to judge whether the subject followed the instructions. In about half of healthy subjects, the vocal folds are not fully closed when they try to follow the 'easy' breath-holding instructions [6,7]. In the humming swallow, the subject is instructed to hum while swallowing instead of holding is breath. Humming induces vocal fold adduction, thereby securing the airway closure to prevent aspiration. The humming swallow has an additional benefit of clearing the food residue from the airway. However, humming decreases lung volume, which could deteriorate the breathing-swallowing coordination.

The second maneuver is the forehead exercise. The head-lifting exercise protocol known as the Shaker exercise consists of isometric and isotonic exercises to strengthen the suprahyoid muscles. However, since the head-lifting exercise requires the physical ability 


\begin{tabular}{|c|c|c|c|c|}
\hline$\sum_{\text {Swallow }}^{\text {test }}$ & $\begin{array}{l}\text { Supraglottic } \\
\text { swallow }\end{array}$ & $\begin{array}{c}\text { Swallow } \\
\text { test }\end{array}$ & $\begin{array}{l}\text { Forehead } \\
\text { exercise }\end{array}$ & $\begin{array}{c}\text { Swallow } \\
\text { Test }\end{array}$ \\
\hline
\end{tabular}

Figure 1: A schema illustrating the study protocol.

to lift the subject's own head, its use for the elderly population may be limited [8]. To overcome this limitation, alternative exercises, aiming at the same goal, have been devised [8-10]. One of the authors (I.F.) has also invented an exercise to strengthen the suprahyoid muscle; in this exercise, the subject firmly attaches his hand on his forehead and strongly pushes his forehead against his hands. Anecdotal evidence suggests that this 'forehead exercise' instantaneously reduced the chance of choking in some patients. However, the mechanisms of the improvement remain unknown. We hypothesize that the forehead exercise maintains the larynx at a higher position, shortens the swallowing latency, and makes the swallow occur earlier in the respiratory cycle, consequently improving the breathing-swallowing coordination.

\section{Materials and Methods}

The protocol was approved by local ethical committees of Hyogo College of Medicine (No. 1715) and Hamamatsu City Rehabilitation Hospital. All subjects gave written informed consent in accordance with the Declaration of Helsinki. Chronic stable patients with dysphasia with Food Intake Level Scale [11] 4-8 (30 subjects, 21 men and 9 women, $75.3 \pm 13.3$ years old) were enrolled in this study. Aspiration or residue in vallecula and/or the pyriform recess was confirmed by video endoscopy or video fluorography in all subjects. All subjects could understand instructions; patients with severe dementia who could not follow the instructions of swallowing maneuvers were excluded from the study.

\section{Study protocol}

Subjects were seated upright on a chair and performed the humming swallow maneuver and the forehead exercise sequentially. The subjects voluntarily swallowed four types of test foods. They swallowed each type of food before, during, and after the humming swallow maneuver, as well as after the forehead exercise (Figure 1). These swallows were monitored by a non-invasive monitoring device [3], and signals were stored in a micro SD card for later analyses.

\section{Instruction to the subjects}

We adopted the humming swallow, a modified version of the supraglottic swallow. Once a test food was put in the subject's oral cavity, the subject was instructed to inhale and then hum to secure the airway clearance before swallowing. Three of 30 subjects could not hum before swallowing and conducted the original version of the supraglottic swallow. Namely, they inhaled breath and hold their breath before swallowing. After swallowing, the subject was instructed to exhale through his mouth.

For the forehead exercise, the subject was instructed to press his palm firmly against his forehead and push his forehead against his hand strongly for 5 seconds, and subsequently push his forehead against his hand for 1 second 5 times.

\section{Test foods}

We adopted the 'Level' system of the Japanese Society of Dysphagia
Rehabilitation [12] to describe the specification of test foods. Test foods we used were Level 0 soft jelly, Level 2 hard jelly, Level 3 purée, and water. The properties (hardness, cohesion, and adhesion) of these test foods were strictly controlled. One piece of test food was approximately $3 \mathrm{~g}$, and water was injected using a syringe. Patients were instructed not to chew test foods and to swallow voluntarily.

\section{Swallowing function evaluation}

The method to monitor swallowing behavior has been described previously [3]. Briefly, respiratory flow was monitored by a nasal cannula-type flow sensor (Flow Nasal Cannula Type A, Atom Medical, Saitama, Japan) and a differential pressure transmitter (KL17, Nagano Keiki Co., Tokyo, Japan). At the same time, laryngeal motion and sound were monitored by a custom-made piezoelectric sensor (Murata Manufacturing, Nagaokakyo, Japan). The following parameters were measured using Matlab software (R2008b, Mathworks, Natick, MA, USA) on a Windows 8.1 computer [4]:

\section{Pause duration}

The duration of deglutition apnea (respiratory pause associated with swallowing).

\section{Swallowing latency}

The time from the onset of respiratory pause to the onset of swallowing reflex. The onset of swallowing reflex was defined as the time point when the speed of the laryngeal elevation reached a maximum.

\section{Old phase}

The timing of the swallow in the respiratory cycle, which is expressed as a number between 0 and 1, where 0 is the beginning of the preceding inspiration and 1 is the mean length of the respiratory cycle [13].

\section{Co-phase}

The time from the swallowing onset to the next inspiration, normalized to the mean length of the respiratory cycle (which was set as 1).

\section{Coordination between breathing and swallowing}

We categorized the breathing-swallowing pattern using two types of parameters: 1) B-SW type, a parameter describing the combination of swallow and the preceding respiratory phase. This type can be either E-SW (expiration-swallow) or I-SW (inspiration-swallow); and 2) SW-B type, a parameter describing the combination of swallow and the following respiratory phase. This can be either SW-E (swallowexpiration) or SW-I (swallow-inspiration).

In addition, 'choking' was identified as coughing immediately after swallowing. No subject exhibited silent aspiration, judging by wet hoarseness.

\section{Statistical analysis}

Changes in parameter values associated with swallowing after maneuvers were compared using repeated-measures analysis of variance (ANOVA), followed by post hoc analyses with the TukeyKramer test. Frequencies of each breathing-swallowing coordination pattern were compared by the chi-square test followed by Haberman's residual analysis. For the humming swallow maneuver, 
Table 1: Relationship between choking and breathing-swallowing parameters.

\begin{tabular}{|c|c|c|c|}
\hline & & Non-Choking & Choking \\
\hline N & & 412 & 18 \\
\hline Old phase & & $1.044 \pm 0.641$ & $1.024 \pm 0.428$ \\
\hline Cophase & & $0.687 \pm 0.508$ & $0.527 \pm 0.331$ \\
\hline Swallow latency & {$[\mathrm{ms}]$} & $302 \pm 573$ & $155 \pm 212$ \\
\hline LRT [3] & {$[\mathrm{ms}]$} & $505 \pm 308$ & $576 \pm 414$ \\
\hline LAD [3] & {$[\mathrm{ms}]$} & $901 \pm 298$ & $928 \pm 351$ \\
\hline Pause duration & {$[\mathrm{s}]$} & $1.044 \pm 0.750$ & $0.935 \pm 0.586$ \\
\hline I-SW frequency & {$[\%]$} & 7.0 & $11.1^{\#}$ \\
\hline SW-I frequency & {$[\%]$} & 13.3 & $22.2^{\#}$ \\
\hline SW-I or I-SW frequency & {$[\%]$} & 19.7 & $33.3^{\#}$ \\
\hline
\end{tabular}

Chi-square $P$ value (\#\# $p<0.0001)$.

Table 2: Changes in breathing-swallowing parameters during and after the modified version of supraglottic swallowing.

\begin{tabular}{|c|c|c|c|c|}
\hline All: 30 patients & & Before & During & After \\
\hline Old phase & & $0.922 \pm 0.523$ & $0.955 \pm 0.471$ & $1.024 \pm 0.573$ \\
\hline Cophase & & $0.673 \pm 0.477$ & $0.783 \pm 0.510$ & $0.642 \pm 0.567$ \\
\hline Swallow latency & [ms] & $236 \pm 447$ & $172 \pm 369$ & $364 \pm 644$ \\
\hline LRT [3] & {$[\mathrm{ms}]$} & $476 \pm 347$ & $506 \pm 300$ & $502 \pm 293$ \\
\hline LAD [3] & [ms] & $917 \pm 298$ & $906 \pm 346$ & $882 \pm 262$ \\
\hline Pause duration & [s] & $1.002 \pm 0.773$ & $0.955 \pm 0.471$ & $1.057 \pm 0.768$ \\
\hline I-SW frequency & [\%] & 9.1 & 6.7 & 5.4 \\
\hline SW-I frequency & [\%] & 10.9 & 16.2 & 13.4 \\
\hline SW-I or I-SW frequency & [\%] & 19.1 & 21.9 & 17.9 \\
\hline Responder: 11 patients & & Before & During & After \\
\hline Old phase & & $0.825 \pm 0.436$ & $1.164 \pm 0.418$ & $0.958 \pm 0.364$ \\
\hline Cophase & & $0.572 \pm 0.310$ & $0.624 \pm 0.360$ & $0.606 \pm 0.302$ \\
\hline Swallow latency & [ms] & $229 \pm 407$ & $95 \pm 243$ & $418 \pm 655$ \\
\hline LRT [3] & [ms] & $553 \pm 339$ & $532 \pm 337$ & $488 \pm 286$ \\
\hline LAD [3] & [ms] & $889 \pm 282$ & $938 \pm 440$ & $907 \pm 285$ \\
\hline Pause duration & {$[\mathrm{s}]$} & $0.937 \pm 0.642$ & $0.888 \pm 0.375$ & $1.081 \pm 0.762$ \\
\hline I-SW frequency & [\%] & 7.7 & 5.6 & 4.9 \\
\hline SW-I frequency & [\%] & 10.3 & 25.0 ** & 7.3 \\
\hline SW-I or I-SW frequency & [\%] & 17.9 & $30.6^{*}$ & 12.2 \\
\hline
\end{tabular}

Chi-square test $P$ value to Before $\left({ }^{*} p<0.05,{ }^{*}{ }^{*} p<0.01\right)$.

swallows before, during, and after the maneuver were compared, and for the forehead exercise, swallows before and after the maneuver were compared. Swallowing kinetics and the breathing-swallowing coordination were further analyzed for the responder group, which was defined as a group of subjects who did not choke after the forehead exercise. All data are presented as the mean \pm standard deviation. $\mathrm{P}$ values were two-sided, and $\mathrm{P}<0.05$ was considered statistically significant.

\section{Results}

Relationship between choking and breathing-swallowing coordination

We observed a total of 430 swallows in 30 patients during the

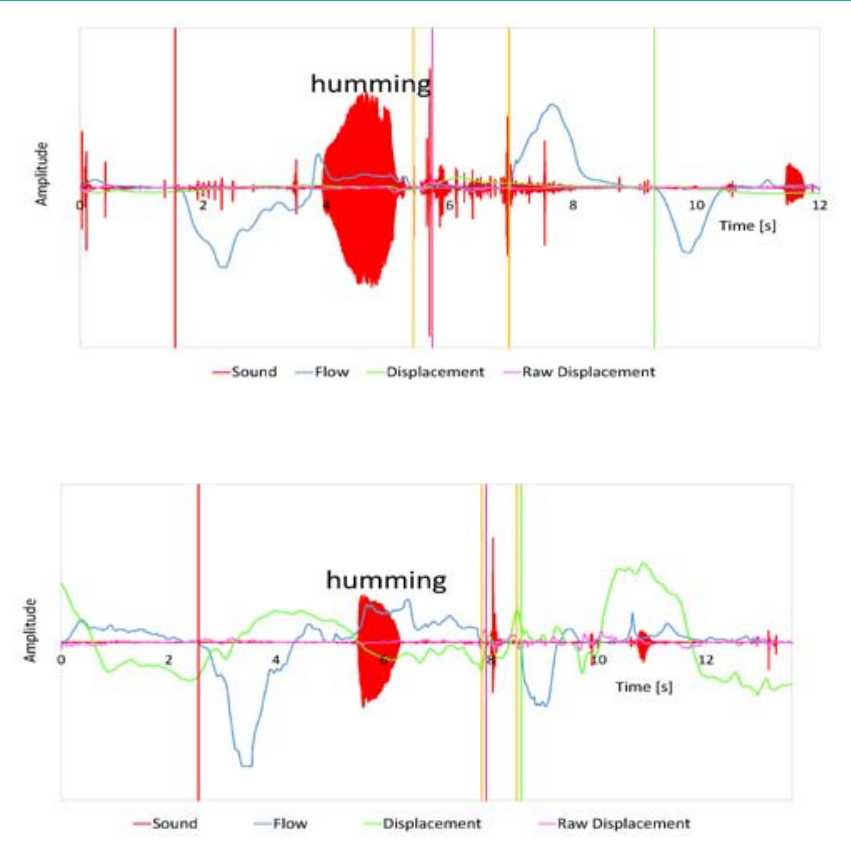

Figure 2: Breathing-swallowing coordination patterns observed during the modified version of supraglottic swallowing. A: A representative case of a swallow with the expiration-swallow-expiration pattern; B: A representative case of a swallow with the expiration-swallow-inspiration pattern. In both traces, respiratory flow, laryngeal sound, and laryngeal displacement signals are shown in blue, red, and green, respectively. A positive shift in the respiratory flow signal represents expiration, and a negative shift represents inspiration. Red, pink, yellow, green horizontal bars represent the onset of the preceding inspiration, the onset of swallowing reflex, the onset and the offset of respiratory pause associated with swallowing, and the onset of the following inspiration, respectively.

study. Among these swallows, 18 swallows were accompanied by choking. As shown in Table 1, the frequency of the I-SW or SW-I pattern was significantly greater in swallows with choking, supporting the hypothesis that swallows with the I-SW or SW-I pattern increase the risk of aspiration.

\section{Breathing-swallowing coordination pattern during the humming swallow maneuver}

The breathing-swallowing patterns before, during, and after the humming swallow maneuver are shown in Table 2. The frequency of inspiration after swallowing (SW-I pattern) increased during the humming swallow compared to the frequency before the maneuver ( $\mathrm{p}=0.0048$ for the responder group). Figure 2 shows representative cases of SW-E pattern and SW-I pattern swallows during the humming swallow. Due to the breath-holding and humming during the humming swallow, swallows with an SW-I pattern took $4.796 \pm$ $2.154 \mathrm{~s}$ to start after the preceding inspiration on an average, resulting in a delayed timing of swallow in the respiratory cycle (old phase = $1.252 \pm 0.482$ ). The frequency of choking was not different between before, during, and after the maneuver.

\section{Changes in swallowing kinetics before the forehead exercise}

Before the forehead exercise, choking was observed in 13 of 30 patients. However, choking persisted in only two patients, suggesting that the forehead exercise had some instantaneous beneficial effects 
Table 3: Changes in breathing-swallowing parameters after the forehead exercise.

\begin{tabular}{|c|c|c|c|}
\hline All: $\mathbf{3 0}$ patients & & Before & After \\
\hline Old phase & & $1.024 \pm 0.573$ & $1.100 \pm 0.886$ \\
\hline Cophase & & $0.642 \pm 0.567$ & $0.626 \pm 0.435$ \\
\hline Swallow latency & [ms] & $364 \pm 644$ & $416 \pm 703$ \\
\hline LRT [3] & [ms] & $502 \pm 293$ & $550 \pm 307$ \\
\hline LAD [3] & [ms] & $882 \pm 262$ & $903 \pm 294$ \\
\hline Pause duration & {$[\mathrm{s}]$} & $1.057 \pm 0.768$ & $1.146 \pm 0.903$ \\
\hline I-SW frequency & [\%] & 5.4 & 7.8 \\
\hline SW-I frequency & {$[\%]$} & 13.4 & 14.6 \\
\hline SW-I or I-SW frequency & [\%] & 17.9 & 22.3 \\
\hline Responder: 11 patients & & Before & After \\
\hline Old phase & & $0.958 \pm 0.364$ & $1.036 \pm 0.985$ \\
\hline Cophase & & $0.606 \pm 0.302$ & $0.585 \pm 0.456$ \\
\hline Swallow latency & [ms] & $418 \pm 655$ & $418 \pm 721$ \\
\hline LRT [3] & [ms] & $488 \pm 286$ & $584 \pm 319$ \\
\hline LAD [3] & [ms] & $907 \pm 285$ & $945 \pm 320$ \\
\hline Pause duration & {$[\mathrm{s}]$} & $1.081 \pm 0.762$ & $1.063 \pm 0.758$ \\
\hline I-SW frequency & [\%] & 4.9 & 8.6 \\
\hline SW-I frequency & [\%] & 7.3 & 14.3 \\
\hline SW-I or I-SW frequency & [\%] & 12.2 & 22.9 \\
\hline
\end{tabular}

on the swallowing function. However, the old phase (the timing of the swallow within the respiratory cycle) and the swallowing latency did not show significant changes (Table 3 ). We further analyzed changes in these parameter values for the responder group in which choking was not observed after the forehead exercise, but none of the parameter values showed significant changes.

\section{Discussion}

The present study confirmed that inspiration before swallowing or after swallowing can increase the risk of aspiration. The humming swallow maneuver increased the frequency of inspiration after swallow. In addition, although the forehead exercise may instantaneously decrease the chance of aspiration, this result could not be explained by its effects on the swallowing latency or the breathing-swallowing coordination.

\section{Humming swallow maneuver}

In the present study, we found that the humming maneuver increased the frequency of inspiration after swallows, especially in responders. We do not know the reason why the responders who did not choke after the forehead exercise more frequently inspired after swallowing. The supraglottic maneuver was invented to secure the closure of the vocal cord, and our modified version intended to clear the airway and to further facilitate the closing of the vocal cord. Therefore, the maneuver should prevent aspiration during swallowing. However, it should be noted that the maneuver prolongs the timing of the swallow in the respiratory cycle and increases the chance of inspiration immediately after swallowing. This maneuver could thereby increase the chance of aspiration after swallowing in patients who have food residues in the pharynx, because inspiration after swallowing may suck the residue into the lower airway. McFarland et al. [14] found that swallows occur at a specific lung volume. Therefore, the humming maneuver might have made some subjects miss the optimal timing of swallowing.

\section{Forehead exercise}

The Shaker exercise, consisting of isometric and isotonic headlifting exercises, augments the opening of the upper esophageal sphincter upon swallowing $[15,16]$ and enhances the thyrohyoid muscle shortening [17]. A preliminary randomized trial demonstrated that the Shaker exercise reduced post-swallow aspiration to a greater degree compared to the traditional swallowing therapy [18]. However, head-lifting may not be feasible for patients with sarcopenia or those in a frail condition. To overcome these problems, a tongue press exercise [8], a jaw opening exercise [9], and the Chin Tuck Against Resistance exercise [10] were developed. These exercises are less strenuous than the Shaker exercise, can be performed in a sitting position, and are reported to be equally effective at strengthening the suprahyoid muscles. The forehead exercise used in the present study is similar to these maneuvers in that it can be performed in a sitting position, and it has the additional advantage of lowering the risk of injuring the temporomandibular joint compared to these maneuvers.

In the present study, although immediate effects of the forehead exercise were present judging from the incidence of choking before and after the maneuver, we could not detect any improvement in the measured values. Further study is needed to elucidate the mechanisms of the immediate effect of the forehead exercise.

\section{Conclusion}

Swallowing kinetics measured by non-invasive techniques were not changed by the humming swallow maneuver or the forehead exercise. The humming swallow maneuver may increase the frequency of inspiration after swallow, so caution should be exercised when this technique is applied to patients with food residues in the pharynx.

\section{Acknowledgments}

The authors wish to thank Tohru Yabe and Kenji Tanaka of Murata Manufacturing Co., Ltd. for providing piezoelectric sensors under an industry-academia cooperative research contract between Hyogo College of Medicine and Murata Manufacturing Co., Ltd.

\section{Conflict of Interest}

Test foods were provided by FoodCare Co., Ltd. Piezoelectric sensors and data loggers were provided by Murata Manufacturing Co., Ltd. under an academia-industry contract between Hyogo College of Medicine and Murata Manufacturing Co., Ltd.

\section{References}

1. Shaker R, Li Q, Ren J, Townsend WF, Dodds WJ, Martin BJ, et al. Coordination of deglutition and phases of respiration: effect of aging, tachypnea, bolus volume, and chronic obstructive pulmonary disease. The American Journal of Physiology. 1992; 263: G750-755.

2. Troche MS, Huebner I, Rosenbek JC, Okun MS, Sapienza CM. Respiratoryswallowing coordination and swallowing safety in patients with Parkinson's disease. Dysphagia. 2011; 26: 218-224.

3. Yagi N, Nagami S, Lin MK, Yabe T, Itoda M, Imai T, et al. A noninvasive swallowing measurement system using a combination of respiratory flow, swallowing sound, and laryngeal motion. Med Biol Eng Comput. 2017; 55: 1001-1017. 
4. Yagi N, Oku Y, Nagami S, Yamagata Y, Kayashita J, Ishikawa A, et al. Inappropriate timing of swallow in the respiratory cycle causes breathingswallowing discoordination. Frontiers in Physiology. 2017; 8: 676.

5. Logemann J. In: Evaluation and Treatment of Swallowing Disorders. San Diego: College Hill Press. 1983: 138-139.

6. Donzelli J, Brady S. The effects of breath-holding on vocal fold adduction: implications for safe swallowing. Archives of otolaryngology--head \& neck surgery. 2004; 130: 208-210.

7. Hirst LJ, Sama A, Carding PN, Wilson JA. Is a 'safe swallow' really safe? Int J Lang Commun Disord. 1998; 33: 279-280.

8. Yoshida M, Groher ME, Crary MA, Mann GC, Akagawa Y. Comparison of surface electromyographic (sEMG) activity of submental muscles between the head lift and tongue press exercises as a therapeutic exercise for pharyngeal dysphagia. Gerodontology. 2007; 24: 111-116.

9. Wada S, Tohara H, lida T, Inoue M, Sato M, Ueda K. Jaw-opening exercise for insufficient opening of upper esophageal sphincter. Arch Phys Med Rehabil. 2012; 93: 1995-1999.

10. Yoon WL, Khoo JK, Rickard Liow SJ. Chin tuck against resistance (CTAR) new method for enhancing suprahyoid muscle activity using a Shaker-type exercise. Dysphagia. 2014; 29: 243-248.

11. Kunieda K, Ohno T, Fujishima I, Hojo K, Morita T. Reliability and validity of a tool to measure the severity of dysphagia: the Food Intake LEVEL Scale. J Pain Symptom Manage. 2013; 46: 201-206.
12. Cichero JA, Lam P, Steele CM, Hanson B, Chen J, Dantas RO, et al. Development of International Terminology and Definitions for TextureModified Foods and Thickened Fluids Used in Dysphagia Management: The IDDSI Framework. Dysphagia. 2017; 32: 293-314.

13. Paydarfar D, Gilbert RJ, Poppel CS, Nassab PF. Respiratory phase resetting and airflow changes induced by swallowing in humans. The Journal of physiology. 1995; 483: 273-288.

14. McFarland DH, Martin-Harris B, Fortin AJ, Humphries K, Hill E, Armeson K. Respiratory-swallowing coordination in normal subjects: Lung volume at swallowing initiation. Respir Physiol Neurobiol. 2016; 234: 89-96.

15. Shaker R, Easterling C, Kern M, Nitschke T, Massey B, Daniels S, et al. Rehabilitation of swallowing by exercise in tube-fed patients with pharyngeal dysphagia secondary to abnormal UES opening. Gastroenterology. 2002; 122: 1314-1321.

16. Shaker R, Kern M, Bardan E, Taylor A, Stewart ET, Hoffmann RG, et al. Augmentation of deglutitive upper esophageal sphincter opening in the elderly by exercise. Am J Physiol. 1997; 272: G1518-1522.

17. Mepani R, Antonik S, Massey B, Kern M, Logemann J, Pauloski B, et al. Augmentation of deglutitive thyrohyoid muscle shortening by the Shaker Exercise. Dysphagia. 2009; 24: 26-31.

18. Logemann JA, Rademaker A, Pauloski BR, Kelly A, Stangl-McBreen C, Antinoja $\mathrm{J}$, et al. A randomized study comparing the Shaker exercise with traditional therapy: a preliminary study. Dysphagia. 2009; 24: 403-411.
Phys Med Rehabil Int - Volume 4 Issue 5 - 2017

ISSN : 2471-0377 | www.austinpublishinggroup.com

Oku et al. (C) All rights are reserved
Citation: Kubo S, Marui M, Yagi N, Nagami S, Hojo K, Kunieda K, et al. Effects of Maneuvers on BreathingSwallowing Coordination and Swallowing Kinetics in Dysphagia Patients. Phys Med Rehabil Int. 2017; 4(5): 1132. 